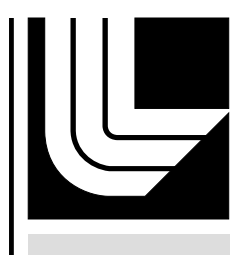

LAW RENCE LIVERMORE N A TIO N A L LABORATORY

\title{
Multiscale Modeling with Carbon Nanotubes
}

A. Maiti

March 1, 2006

Microelectronics Journal 
This document was prepared as an account of work sponsored by an agency of the United States Government. Neither the United States Government nor the University of California nor any of their employees, makes any warranty, express or implied, or assumes any legal liability or responsibility for the accuracy, completeness, or usefulness of any information, apparatus, product, or process disclosed, or represents that its use would not infringe privately owned rights. Reference herein to any specific commercial product, process, or service by trade name, trademark, manufacturer, or otherwise, does not necessarily constitute or imply its endorsement, recommendation, or favoring by the United States Government or the University of California. The views and opinions of authors expressed herein do not necessarily state or reflect those of the United States Government or the University of California, and shall not be used for advertising or product endorsement purposes. 


\title{
Multiscale Modeling with Carbon Nanotubes
}

\author{
Amitesh Maiti ${ }^{\dagger}$ \\ Lawrence Livermore National Laboratory, University of California, Livermore, \\ CA 94551, USA
}

\begin{abstract}
Technologically important nanomaterials come in all shapes and sizes. They can range from small molecules to complex composites and mixtures. Depending upon the spatial dimensions of the system and properties under investigation computer modeling of such materials can range from equilibrium and nonequilibrium Quantum Mechanics, to force-field-based Molecular Mechanics and kinetic Monte Carlo, to Mesoscale simulation of evolving morphology, to Finite-Element computation of physical properties. This brief review illustrates some of the above modeling techniques through a number of recent applications with carbon nanotubes: nano electromechanical sensors (NEMS), chemical sensors, metal-nanotube contacts, and polymernanotube composites.
\end{abstract}

Keywords: DFT, Molecular Modeling, NEGF, Mesoscale modeling, Nanotubes, Sensors, Nanocomposites

\footnotetext{
${ }^{\dagger}$ E-mail: maiti2@llnl.gov
} 


\section{Introduction}

More than fifteen years after its initial discovery, carbon nanotubes (CNTs) [1] continue to be one of the hottest research areas in all of science and engineering. The interest is driven by the possibility of several commercial applications [2-6], including: Field Emission-based Flat Panel displays, transistors, quantum dots, hydrogen storage devices, structural reinforcement agents, chemical and electromechanical sensors, nanoscale manipulators, probes, and tweezers. At the same time, the highly regular atomic structure of carbon nanotubes and the large degree of structural purity makes it accessible to accurate computer modeling using a variety of theoretical techniques. In fact, ever since the discovery of CNTs it has provided a fertile ground for theoretical simulations and analysis. The prediction of the dependence of CNT's electronic structure on its chirality [7-9] came within a year of the initial experimental discovery [1]. Since then there have been a huge number of theoretical investigations [10-18] of growth mechanisms, structure and energetics of topological defects, mechanical and electrical response to various kinds physical perturbation, field-emission from tips of metallic CNTs, electronic effects of doping and gas adsorption, chemical reactivity, interaction with polymers, capillary effects, CNT-metal contacts, H- and Li-storage, thermal conductivity, encapsulation of organic as well as inorganic material, optical properties, as well as novel quantum effects. Computational approaches used in the above work include solving diffusion equations, QM simulations (DFT, tight-binding, and semi-empirical methods), classical molecular dynamics, kinetic Monte Carlo, Genetic algorithms, and Green's-function-based electronic transport theory. This paper illustrates several of the above theoretical techniques through a few recent modeling studies of CNTs. In the following, we provide a brief overview of the theoretical techniques, which is followed by separate sections detailing each application example.

\section{Theoretical Techniques: A brief overview}

\subsection{Density Functional Theory (DFT)}


In principle, all chemical information of any nanostructure can be obtained if one could solve the Schrödinger wave equation for all relevant electrons of the system. Ideally, all one should require as an input are the positions and element identity of all atoms describing the nanostructure. Such computational methods are called ab initio or First-Principles methods [19]. The Schrödinger equation of an interacting system of electrons cannot be solved analytically, and involves a number of physically motivated approximations and clever numerical tricks. The most well accepted formalisms can be broadly classified into two types: those based on the Hartree-Fock method [20], and those based on the Density Functional Theory (DFT) [21]. Due to better scaling with the number of electrons, DFT is quickly becoming the First-Principles technique of choice in technologically important problems. DFT is based on a theorem due to Hohenberg and Kohn [22], which states that all ground state properties are functions of the total electronic charge density $\rho(\boldsymbol{r})$. The total energy of an electron gas can be formalistically written as a sum of the kinetic, potential (electrostatic), exchange and correlation energies. A practical implementation of this formalism into computer programs was made possible by the local density approximation (LDA) of Kohn and Sham [23], which recast the many-electron problem into a problem of single electrons moving in an average field of the other electrons and ions. The basic formalism has stood the test of time, although important subsequent developments on gradient corrections to the LDA and the exchange-correlation functional have increased the accuracy of DFT significantly. There are several different DFT codes available commercially, differing primarily in the choice of the basis functions in which the electronic wave functions are expanded, and the scheme of integration. For the work reported here, we used the DFT code $\mathrm{DMol}^{3}$ [24]. In the present work all electrons in the system were considered explicitly, and the electronic wave functions were expanded in a doublenumeric polarized (DNP) basis set. The calculations employed a "medium" integration grid, and a gradient-corrected exchange-correlation functional due to Perdew, Burke, and Ernzerhof [25].

\subsection{Classical Molecular Mechanics}


Some of the applications discussed here, especially electromechanical sensing and polymerCNT composites involve long nanotube structures with several hundred to a few thousand atoms. First-principles DFT calculations on systems of such size is prohibitively expensive. An explicit treatment of electrons is also unnecessary in situations where no chemical bonds are made or broken during the process of interest. In such cases one uses classical molecular mechanics (MM) employing interatomic interactions or force fields, which are parameterized analytical functions of the atomic positions only [26]. For the polymer-CNT composite system, we used a class II force field COMPASS [27], developed primarily for condensed organic systems. For NEMS applications, on the other hand, a metallic AFM tip was modeled by a needle made up of Li atoms. Interactions in such a system were described by the Universal force field (UFF) [28], which is rules-based and has a broader coverage of the periodic table. In order to explore possible chemical rebonding in a localized region one often cuts a small model out of the original structure, and relaxes it with a quantum mechanical $(\mathrm{QM})$ method of choice. Such a hybrid QM/MM method using $\mathrm{DMol}^{3} / \mathrm{UFF}$ was employed in the NEMS application, as discussed below in section 4.1.

\subsection{Electronic Transport modeling - non-equilibrium Green's function}

Electromechanical and chemical sensors typically operate by changing electrical conductance of the active device when subjected to a mechanical perturbation or upon the absorption of a chemical species. Mesoscopic electron transport through molecular wires is best described by an energy-dependent transmission function $T(E)$, which strongly depends on the (discrete) electronic levels of the molecular wire (in our case, a nanotube), the levels in the (usually metallic) leads or electrodes, and broadening of the electronic levels in the wire due to chemical coupling to the electrodes [29-33]. Such physics is most conveniently described under the formalism of nonequilibrium Green's function (NEGF). The starting point is the Green's function of an isolated system at an energy $E$, which is defined by the equation:

$$
\left(E \cdot S_{i j}-H_{i j}\right) G^{R, j k}=\delta_{i}^{k}
$$


where $\delta_{i}^{k}$ is the Kronecker delta, and $S_{i j}=\langle i| j>$ and $H_{i j}=\langle i|H| j>$ are the overlap and Hamiltonian matrix elements between electronic states $i$ and $j$ respectively. However, we are interested in systems in which a nanoscale region is coupled with two semi-infinite electrodes at the two ends (the so called two-probe system). In such a system, the coupling to the electrodes (mathematically expressed in terms of the so-called self-energy matrices $\Sigma$ ) modifies equation (2.1) to the form:

$$
\left(E \cdot S_{i j}-H_{i j}-\Sigma_{L, i j}-\Sigma_{R, i j}\right) G^{R, j k}=\delta_{i}^{k},
$$

where $\Sigma_{L, R}$ are the retarded self-energies of the left and the right semi-infinite contacts. The transmission at each energy is then found [29-33] from the equation:

$$
T(E)=G^{R, i j} \Gamma_{L, j k} G^{A, k l} \Gamma_{R, l i}
$$

where $\Gamma_{L, R}=i\left(\Sigma_{L, R}^{R}-\Sigma_{L, R}^{A}\right)$ are the couplings to the left and right leads and the superscripts $R$ and $A$ represent retarded and advanced, respectively. Finally, the total conductance of the tube is computed using Landauer-Büttiker formula [29-33]:

$$
G=\frac{2 e^{2}}{h} \int_{-\infty}^{\infty} T(E)\left(-\frac{\partial f_{o}}{\partial E}\right) d E
$$

where $f_{o}(E)$ is the Fermi-Dirac function. The electronic states in the system, and more specifically the various matrix elements can be obtained either from first-principles or semi-empirical (e.g., tight-binding) quantum mechanical treatments. Also, if the interest is to investigate changes to the intrinsic electrical conductance of the nanotube, a common trick is to define "ideal" semi-infinite contacts based on defect-free unstrained pieces of the pristine nanotube. Such a procedure bypasses the necessity to model explicit metallic contacts, which is likely to involve additional chemical complexities on the top of extra computational burden.

\subsection{Mesoscale modeling - dissipative particle dynamics (DPD)}


Many interesting problems in soft matter occur at length and time-scales sandwiched between the atomistic scale and the macroscopic continuum. Examples include polymer adsorption, polymer-surfactant interaction, microphase separation of block copolymers, formation and coalescence of droplets in emulsion, transport through living cells, and formation of polymer networks, to name just a few. Such systems can involve spatial inhomogeneities over length-scales ranging between 1-1000 nm, and exhibit dynamical phenomena over time-scales of $1 \mathrm{~ms}$ or greater. Problems at such length and time-scales cannot be directly addressed by traditional atomic-level molecular dynamics, or by conventional finite-element approaches that usually deal with phenomena at longer length scales. Rather, one needs to take recourse to computational techniques at the intermediate scale, called the mesoscale. Almost every day colloid and polymer scientists find examples of novel mesoscale morphologies and explore possible applications ranging from traditional high-impact polymer materials, high-performance elastomers, advanced drug-delivery capsules and biochips, artificial skin and smart gels, contact lens, to polymer displays. Over the last few years several different approaches have been developed to address problems at the mesoscale, which could be broadly classified as either particle-based or densitybased. In order to study polymer-nanotube composites, we have made use of a particle-based method called Dissipative Particle Dynamics (DPD) [34].

In DPD, one represents a group of atoms (typically entire functional groups) by a single bead, thereby substantially reducing the number of particles to be simulated. The positions and velocities of the spherical beads are propagated by standard integrators as in regular MD methods and thermally equilibrated through a Langevin thermostat. But rather than interact through LennardJones forces the beads feel a simple soft pair-wise conservative potential which embodies the essential chemistry of the system. This force is short range and has a simple analytic form resulting in fast computation per time-step. More importantly it provides an effective time-step of several picoseconds, 3-4 orders of magnitude larger than typical time-steps employed in a MD simulation. 
In the basic DPD formalism, the force $\vec{f}_{i}$ on bead $i$ contains three parts, each of which is pairwise additive:

$$
\vec{f}_{i}=\sum_{j}^{\prime}\left(\vec{f}_{i j}^{c}+\vec{f}_{i j}^{D}+\vec{f}_{i j}^{R}\right) .
$$

The chemistry of mixing and segregation is governed by the term $f_{i j}^{c}$, which is modeled as a soft repulsion between beads $i$ and $j$. It is a function of the relative separation between the beads and act along the line joining the two beads, thus being conservative (i.e. momentum conserving). Groot and co-workers [34-36] have established a relation between the interaction parameters describing the $f_{i j}{ }^{c}$ term and the Flory-Huggins $\chi$-parameter [37, 38] for beads $i$ and $j$. Basically, the repulsion is proportional to an interaction parameter $\boldsymbol{a}_{i j}$, and it is the excess interaction $\Delta \bar{a}=\left(\bar{a}_{A B}-\bar{a}_{A A}\right)$ that determines whether components $\mathrm{A}$ and $\mathrm{B}$ will mix or segregate. The other two terms $f^{D}$ and $f^{R}$ are dissipative and random forces respectively, which in tandem act as a thermostat and result in fast equilibration to the Gibbs-Boltzmann canonical ensemble [39].

In the basic Groot-Warren theory, all the three interaction terms described above have the same finite range $R_{c}$, which sets the basic length-scale of the system, and is defined as the side of a cube containing an average number of $\bar{\rho}$ beads. Therefore $R_{c}=\left(\bar{\rho} V_{b}\right)^{1 / 3}$, where $V_{b}$ is the volume of a bead. One can think of $\bar{\rho}$ as a dimensionless bead-density, related to the average beaddensity $\rho=1 / V_{b}$ by the equation $\bar{\rho}=\rho R_{c}{ }^{3}$. Even in a heterogeneous system consisting of several different species, the basic assumption is that all bead-types (each representing a single species) are of the same volume $V_{b}$. This assumption is necessary in order to conform to the Flory-Huggins $\chi$-parameter theory.

In addition to the three forces described above, polymeric systems also require extra springlike interactions to describe covalent "bonding" between consecutive beads in a polymeric chain. Hookean spring potentials have traditionally been used [34]:

$$
E_{i j}=\frac{1}{2} k^{B} \bar{r}_{i j}^{2},
$$


where the energy is dependent only on the separation of the beads $\bar{r}_{i j}$ (expressed in reduced units, i.e., $\left.\bar{r}_{i j}=r_{i j} / R_{c}\right)$ and leads to a force $F_{i j}=k^{B} \bar{r}_{i j}$, where $k^{B}$ is the force constant. This scheme has been shown to successfully reproduce the end-point distribution and exhibit the correct scaling laws for chains as short as $L=10$, although a microscopic theory mapping the detail of the polymer to the DPD strings has not so far been developed. For $k^{B}$ it appears sufficient to use a value that does not allow excessive stretching of the bond; $k^{B}=4.0$ has often been used and was used here for bonds in all polymer chains in the polymer-nanotube composite models. It should be noted that although the minimum bond energy is achieved for $r_{i j}=0$, overlapping of the beads is prevented by the soft repulsive bead-bead interaction, which works against this. There are no 1-2 or 1-3 exclusions from the soft repulsive interaction for bonded DPD beads.

In an extension to our previous work [40], and in order to represent the bending rigidity inherent to carbon nanotubes, we also added an angle dependent potential. This term is represented by a standard cosine bond angle expression as follows [41]:

$$
E_{i j k}=\frac{1}{2} k^{\theta}\left(1+\cos \theta_{i j k}\right) .
$$

Once the mesoscale morphologies are obtained by DPD, one could compute important physical properties of the nanocomposites by representing the morphology on a numerical grid, and perform finite element calculations [42-44]. For each of the grid elements such an approach specifies the fraction of each phase contained in that particular position, and uses proper combination rules to compute specific properties. Technical details can be found in Gusev et al. [42]. For CNT-polymer composites the properties of most interest are electrical and thermal conductivities.

\section{Carbon nanotube (CNT) basics}

A CNT can be geometrically thought of as a graphite sheet rolled into a seamless cylinder. A necessary condition for the cylinder to be seamless is that upon rolling, a graphite lattice point $\left(n_{l}\right.$, 
$n_{2}$ ) coincides with the origin $(0,0)$. Thus, if $\boldsymbol{a}_{1}$ and $\boldsymbol{a}_{2}$ are the two lattice vectors of graphite, the CNT circumference is equal to the length of the vector $\left(n_{1} \boldsymbol{a}_{1}+n_{2} \boldsymbol{a}_{2}\right)$, while the CNT chiral angle $\theta$ is defined as the angle between vectors $\left(n_{1} \boldsymbol{a}_{1}+n_{2} \boldsymbol{a}_{2}\right)$ and $\boldsymbol{a}_{1}$. With the choice of lattice vectors as in Fig. 1(a), the chiral angle and diameter of a CNT are given respectively by the formulas:

$$
\begin{aligned}
& \theta=\tan ^{-1}\left[\sqrt{3} n_{2} /\left(2 n_{1}+n_{2}\right)\right], \quad \text { and } \\
& d=a \sqrt{\left(n_{1}^{2}+n_{1} n_{2}+n_{2}^{2}\right)} / \pi,
\end{aligned}
$$

where $a=\left|\boldsymbol{a}_{1}\right|=\left|\boldsymbol{a}_{2}\right| \sim 2.45 \AA$ is the lattice constant of graphite. The CNT diameter and chirality, and therefore its atomic geometry is completely specified by the two integers $\left(n_{1}, n_{2}\right)$, which are referred to as the chiral indices of the CNT. Due to the symmetry of the graphite lattice, a nanotube of any arbitrary chirality can be defined in the range $n_{1} \geq n_{2} \geq 0$ and $n_{1}>0$, which implies that the chiral angle $\theta$ for all CNTs lies between 0 and $30^{\circ}$. CNTs with the extreme chiral angles of 0 and $30^{\circ}$ have special names: a CNT with $\theta=0$ (i.e. $n_{2}=0$ ) is called zigzag, while a CNT with $\theta=30^{\circ}\left(n_{1}=n_{2}\right)$ is called armchair. The names armchair and zigzag simply reflect the shape of the open edges of these CNTs (Figs. 1(b, c)). CNTs with any other chiral angles (i.e., $0<$ $\theta<30^{\circ}$ ) are called chiral.

Armchair and zigzag CNTs possess small periodic repeat lengths along the nanotube axis, the repeat-length being only $a(\sim 2.45 \AA)$ for armchair tubes and $\sqrt{3} a(\sim 4.24 \AA)$ for zigzag tubes. Chiral CNTs on the other hand can possess very long periodic repeat lengths depending on the ratio of its chiral indices. Thus, electronic structure calculations, especially those employing firstprinciples Quantum Mechanics (QM) with periodic boundary conditions, are rarely performed on chiral tubes. However, this does not turn out to be a serious limitation. Since a CNT is just a rolled-up graphite sheet, one can obtain a good approximation to the CNT electronic structure simply by applying an appropriate boundary condition to the electronic structure of a graphite sheet, with a small perturbation due to the finite cylindrical curvature of the CNT surface. The boundary condition for a CNT with chiral indices $\left(n_{1}, n_{2}\right)$ corresponds to the coincidence of the $\left(n_{1}, n_{2}\right)$ lattice point of graphite with the origin $(0,0)$. It has been known for some time that a 
single sheet of graphite (also known as graphene) is neither a semiconductor nor a metal, but a semi-metal [45] (i.e., a zero-bandgap semiconductor). This peculiarity implies that the electronic states of graphene are very sensitive to additional boundary conditions that a CNT mandates. Taking into account small effects due to curvature, such boundary conditions lead to the important result $[7-9,46-48]$ that all armchair tubes are metallic; CNTs with $n_{1}-n_{2}=3 n(n=$ any positive integer), which includes the $(3 n, 0)$ zigzag tubes as a special class, are quasi-metallic (small bandgap $\sim 10 \mathrm{meV}$ or less, arising from curvature effects); and CNTs with $n_{1}-n_{2} \neq 3 n$ are semiconducting, with a bandgap decreasing as $1 / d$ as a function of tube diameter $d$ (thereby converging to the zero bandgap of graphite in the limit $d \rightarrow \infty$ ). Experimental measurements are often not able make the distinction between metallic and quasi-metallic tubes because of the presence of contact resistance and thermal effects. Thus for simplicity, experimentalists often classify CNTs as either metallic or semiconducting, and we follow the same convention here.

Chirality-dependent electrical conductance of CNTs is only one of its many fascinating properties $[2-6,13-17]$ :

- Single- or multi-walled CNTs are exceptionally strong, could possess a Young's modulus as high as 1.2 Terapascal, six times the modulus of steel

- CNTs have very high thermal conductivity, as high as $6 \times 10^{3} \mathrm{~W} /(\mathrm{m} . \mathrm{K})$ for isolated tubes [49].

- CNTs are elastic to the highest degree, and do not display plasticity behavior even under large deformation including stretching, bending, or twisting [13].

- Metallic CNT are 1-dimensional quantum conductors where electrons travel ballistically: there is no heat dissipation along the length of the CNT. All dissipation occurs at the contacts.

- CNTs can have huge aspect ratio (i.e., length to diameter ratio), as large as $10^{5}$ or even more. Field-emission of electrons can, therefore, be induced from the tip of long metallic CNTs in the presence of moderate electric fields.

- Depending on its chiral indices, metallic CNTs can undergo metal-to-semiconductor transition under small tensile or torsional strain. 
- With a magnetic field parallel to its axis, A CNT can exhibit the Aharonov-Bohm effect.

- Atoms/molecules can be enclosed inside a CNT.

- A CNT can be doped both p-type and (to a lesser degree) n-type.

Before moving onto applications, it should be noted that CNTs come in two forms, i.e., one consisting of a single cylindrical layer (single-walled nanotubes, or SWNTs) and one comprising several concentric layers (multi-walled nanotubes, or MWNTs). The inter-layer interaction within MWNTs is primarily van der Waals type, and nearly all interesting properties are dominated by the individual layers. Therefore, for this paper, we confine all discussions on CNTs to SWNTs.

\section{Application examples}

\subsection{Electromechanical sensors}

Interest in the application of carbon nanotubes as electromechanical sensors got a significant boost from the pioneering experiment of Tombler et al [50], in which the middle part of a segment of a metallic nanotube suspended over a trench was pushed with an AFM tip. Beyond a deformation angle of $\sim 10^{\circ}$ the electrical conductance of the tube dropped by more than two orders of magnitude. The effect was found to be completely reversible, i.e., through repeated cycles of AFM-deformation and tip removal, the electrical conductance displayed a cyclical variation with constant amplitude.

The drop in conductance in the AFM-deformed tube was much higher than the computationally predicted values for tubes bent under mechanical duress. Such calculations, using both tight-binding [51] and semi-empirical Extended-Hückel type approaches [52] concluded that even under large bending angles the reduction in electrical conductance was less than an order of magnitude. For AFM-deformed nanotubes, in contrast, $\mathrm{O}(\mathrm{N})$ tight-binding calculations [53] show that beyond a critical deformation several C-atoms close to the AFM tip become $\mathrm{sp}^{3}$-coordinated. The $\mathrm{sp}^{3}$ coordination ties up delocalized $\pi$-electrons into localized $\sigma$-states. This would naturally explain the large drop in electrical conductivity, as verified by explicit transport calculations. 
Realizing that an AFM-deformed tube also undergoes tensile stretching (see Fig. 2), and a stretched tube belonging to certain chirality class can undergo significant changes in electrical conductance upon stretching, we carried out independent calculations to check the above $\mathrm{sp}^{3}$ coordination idea. The smallest models of CNTs necessary in such simulations typically involve a few thousand atoms, which makes First-Principles Quantum Mechanics simulations unfeasible. Therefore, as described below, we carried out a combination of first-principles DFT and classical molecular mechanics to investigate structural changes in a CNT under AFM-deformation. Bond reconstruction, if any, is likely to occur only in the highly deformed, non-straight part of the tube close to the AFM-tip. For such atoms, we used a DFT-based quantum mechanical description ( $\sim 150$ atoms including AFM-tip atoms), while the long and essentially straight part away from the middle was described accurately using the Universal force field (UFF) [28].

Because of known differences in electronic response of zigzag and armchair tubes to mechanical deformation, we studied a $(12,0)$ zigzag and a $(6,6)$ armchair tube, each consisting of 2400 atoms. The AFM tip was modeled by a 6-layer deep 15 -atom Li-needle normal to the (100) direction, terminating in an atomically sharp tip (see Fig. 3(a)). To simulate AFM-tip-deformation, the Li-needle was initially aimed at the center of a hexagon on the bottom-side of the middle part of the tube. The Li-needle tip was then displaced by an amount $\delta$ toward the tube along the needleaxis, resulting in a deformation angle $\theta=\tan ^{-1}(2 \delta / L), L$ being the unstretched length of the tube. At each end of the tube, a contact region was defined by a unit cell plus one atomic ring (a total of 36 and 60 atoms for the armchair and the zigzag tube respectively). The whole tube was then relaxed by UFF keeping the needle atoms and the end contact regions of the tube fixed. The contact region atoms were fixed in order to simulate an ideal undeformed semi-infinite carbon nanotube lead, and to ensure that all possible contact modes are coupled to the deformed part of the tube. Following the UFF relaxation, a cluster of $132 \mathrm{C}$-atoms for the $(6,6)$ tube, and a cluster of 144 C-atoms for the $(12,0)$ tube were cut out from the middle of the tubes. These clusters (plus the AFM-tip atoms), referred to below as the QM clusters, were further relaxed with the DFT-code $\mathrm{DMol}^{3}$ [24], with the end atoms of the cluster plus the Li-tip atoms fixed at their respective classical positions. 
Fig. 3(b) displays the tip-deformed QM-cluster for the $(6,6)$ tubes at the highest deformation angle of $25^{\circ}$ considered in these simulations. Even under such large deformations, there is no indication of $\mathrm{sp}^{3}$ bonding (the same is true for a $(12,0)$ tube), and the structure was very similar to what was previously observed for a $(5,5)$ tube [54]. The absence of $\mathrm{sp}^{3}$ coordination is inferred based on an analysis of nearest-neighbor distances of the atoms with the highest displacements, i.e., the ones closest to the Li-tip. Although for each of these atoms the three nearest neighbor C-C bonds are stretched to between 1.45-1.75 $\AA$, the distance of the fourth neighbor, required to induce $\mathrm{sp}^{3}$ coordination is greater than $2.2 \AA$ for all tubes in our simulations. The electronic charge density in the region between a C-atom and its fourth nearest neighbor is negligibly small, and none of the $\mathrm{C}-\mathrm{C}-\mathrm{C}$ angles between bonded atoms in the vicinity of the tip deviates by more than a few degrees from $120^{\circ}$, suggesting that the C-atoms near the AFM-tip essentially remain $\mathrm{sp}^{2}$ coordinated.

Following structural relaxation of the CNTs as described above, we computed the transmission and conductance through the deformed CNT using a NEGF formalism, as alluded to in section 2.3. The electrons were described using a nearest-neighbor $\mathrm{sp}^{3}$-tight-binding Hamiltonian in a non-orthogonal basis. The parameterization scheme explicitly accounts for effects of strain in the system through a bond-length-dependence of the Hamiltonian and the overlap matrices $H_{i j}$ and $S_{i j}$, as in Ref [55].

Our results indicate that the conductance remains essentially constant for the $(6,6)$ armchair tube up to deformation as large as $25^{\circ}$. However, for the $(12,0)$ tube the conductance drops by a factor of $\sim 0.3$ at $15^{\circ}$, two orders of magnitude at $20^{\circ}$, and 4 orders of magnitude at $\theta=25^{\circ}$. Since $\mathrm{sp}^{3}$ coordination could be ruled out, the only logical explanation of the observed behavior could be due to stretching. We verified that by computing conductance changes due to pure tensile stretching and comparing the results with that of AFM-deformed tubes (Fig. 4). It should also be noted that the $(12,0)$ tube displays only a $70 \%$ drop in electrical conductance at $\theta=15^{0}$, while the tube in ref. [50] underwent more than two orders of magnitude drop. This can be explained by the fact that the $(12,0)$ tube has a diameter of only $\sim 1 \mathrm{~nm}$, while the experimental tube was of 
diameter $\sim 3 \mathrm{~nm}$. A $(36,0) \mathrm{CNT}$, with diameter similar to the experimental tube, indeed displays a much higher drop in electrical conductance (Fig. 4).

In order to explain the differences in conductance drops of the armchair $(6,6)$ and the zigzag $(12,0)$ tubes as a function of strain, we turn to the literature where a considerable amount of theoretical work already exists [56-61]. An important result [60] is that the rate of change of bandgap as a function of strain depends on the CNT chiral angle $\theta$, more precisely as proportional to $\cos (3 \theta)$. Thus, stretched armchair tubes $\left(\theta=30^{\circ}\right)$ do not open any bandgap, and always remain metallic. On the other hand, a metallic $(3 n, 0)$ zigzag tube $(\theta=0)$ can open a bandgap of $\sim 100$ meV when stretched by only $1 \%$. This bandgap increases linearly with strain, thus transforming the CNT into a semiconductor at a strain of only a few percent. In general, all metallic tubes with $n_{1}-n_{2}=3 n(n>0)$ will undergo the above metal-to-semiconductor transition, the effect being the most pronounced in metallic zigzag tubes. An experiment as in ref. [50] is, thus, expected to show a decrease in conductance upon AFM-deformation for all nanotubes except the armchair tubes. Researchers are also beginning to explore electrical response of squashed CNTs [62-64], where $\mathrm{sp}^{3}$ coordination is a possibility.

In addition to the above results for metallic CNTs, theory also predicts that [60] for semiconducting tubes $\left(n_{1}-n_{2} \neq 3 n\right)$, the bandgap can either increase (for $n_{1}-n_{2}=3 n-2$ ) or decrease (for $n_{1}-n_{2}=3 n-1$ ) with strain. These results have recently prompted more detailed experiments on a set of metallic and semiconducting CNTs deformed with an AFM-tip [65], as well as on CNTs under experimental tensile stretch [66]. Commercial applications from such work could lead to novel pressure sensors, transducers, amplifiers, and logic devices [67].

\subsection{Metal-CNT contact}

Measured conductance through a nanodevice depends strongly on the contact resistance of the metal electrodes, and CNT-based electronic devices are no exception. Besides, CNTs interacting with metal nanoparticles are gaining considerable interest as sensing materials, catalysts, in the 
synthesis of metallic nanowires, as well as in nanoelectronics applications as Field-EffectTransistor (FET) devices. A systematic study of electron-beam-evaporation-coating of suspended CNT with various metals reveals that the nature of the coating can vary significantly depending upon the metal [68]. Thus, Ti, Ni and Pd form continuous and quasi-continuous coating, while Au, $\mathrm{Al}$ and $\mathrm{Fe}$ form only discrete particles on the CNT surface. In fact, $\mathrm{Pd}$ is a unique metal in that it consistently yields ohmic contacts to metallic nanotubes [69], and zero or even negative Schottky barrier at junctions [70] with semiconducting CNTs for FET applications. The Schottky barrier (for $p$-channel conductance) could, in principle, be made even lower if a higher work function metal, e.g., Pt is used. Unfortunately, Pt appears to form non-ohmic contacts to both metallic and semiconducting CNTs with lower p-channel conductance than Pd-contacted junctions.

The computed interaction energy of a single metal atom on a CNT [71] follows the trend $E_{b}(\mathrm{Ti})>E_{b}(\mathrm{Pt})>E_{b}(\mathrm{Pd})>E_{b}(\mathrm{Au})$. These trends would suggest that Ti sticks the best to the CNT and $\mathrm{Au}$ the worst, in good agreement with experiment. However, it does not explain why Pt consistently makes worse contacts than $\mathrm{Pd}$, and why $\mathrm{Ti}$, in spite of its good wetting of a CNT surface, yields Ohmic contacts only rarely [69]. A detailed investigation of the metal-CNT contact at full atomistic detail is a significant undertaking, and is likely beyond the realm of today's firstprinciples Quantum Mechanics codes. Nevertheless, as a first attempt, it is instructive to look into the interactions of CNTs with metallic entities beyond single atoms.

To this end, we carried out binding energy calculations of metallic monolayers, multilayers, and 13-atom clusters with a sheet of graphite, which is a representative of wide-diameter CNTs. In addition, the interaction of a semiconducting $(8,0)$ tube with flat metallic surfaces was also studied. Three metals were considered for concreteness - Au, Pt, and Pd. Calculations were performed with the DFT code $\mathrm{DMol}^{3}$ [24]. Details are given in ref. [72]. We only summarize the main results below:

i) For isolated $\mathrm{Au}, \mathrm{Pd}$, and $\mathrm{Pt}$ atoms on a sheet of graphene, the respective binding energies are $0.30,0.94$ and $1.65 \mathrm{eV}$ respectively, i.e., in the same order as previous computed values on a $(8,0)$ 
CNT [71]. The binding sites are also quite similar, although the binding energies to graphene are $40 \%$ smaller than to the CNT, whose finite curvature imparts higher reactivity.

ii) For monolayer or multilayer of metal atoms on graphene, most of the metal binding arises from metal-metal interaction rather than metal-graphene interaction. This is due to high cohesive energies of the metals in the bulk crystalline state. If only the metal-graphene part of the interaction is considered, the binding for Pt falls rapidly with layer thickness, and is less than that of Pd for 3-layer films, perhaps indicating possible instability of Pt films beyond a certain thickness. This is likely due to much higher cohesive energy of Pt as compared to Pd. For Au, isolated atoms as well as films interact very weakly with graphene, in agreement with experimentally observed poor wetting properties.

iii) 13-atom Pd clusters bind more strongly to the graphene surface than a 13-atom Pt cluster. The Pd cluster, in particular, gets significantly distorted from its ideal icosahedral geometry. Spin might play an important role in such binding calculations, and requires a more careful analysis.

iv) We predict a critical cluster size of a few tens of metal atoms below which the metal should wet a graphene (and therefore CNT) surface uniformly, and above which non-uniform clustering is likely. Using a simple model we show that the critical cluster size for Pt is smaller than that for Pd, implying higher propensity of Pt to form a non-uniform coating unless it is deposited in the form of ultrafine nanoparticles.

v) Finally, CNTs placed on flat Pt or Pd surface can form direct covalent bonds to the metal, which, along with the resulting deformation in tube cross-section might alter its electronic properties and impact performance of electronic devices based on such geometry. Interaction with a Au surface is weak, and the CNT cross-section remains circular. This phenomenon is illustrated in Fig. 5.

\subsection{Chemical sensors}


Of the projected electronics-based application areas of CNTs, gas sensors appear to show a lot of commercial promise. Detection of gas molecules such as $\mathrm{NO}_{2}, \mathrm{O}_{2}, \mathrm{NH}_{3}, \mathrm{~N}_{2}, \mathrm{CO}_{2}, \mathrm{H}_{2}, \mathrm{CH}_{4}, \mathrm{CO}$, or even water is important for monitoring environmental, medical or industrial conditions. Recently, it was reported [73-77] that the measured electrical conductance of a semiconducting $\mathrm{CNT}$ at room temperature increases by three orders of magnitude upon exposure to $0.2 \%$ of $\mathrm{NO}_{2}$, and decreases by two orders of magnitude upon exposure to $1 \%$ of $\mathrm{NH}_{3}$. Exposure to $\mathrm{O}_{2}$ also appears to consistently increase the electrical conductance, although the effect is not as dramatic as for $\mathrm{NO}_{2}$.

Considering the fact that the CNT used in the experiment of ref. [73] was effectively $p$-type (i.e. holes were the majority carriers), the observed behavior of conductance changes can be rationalized through a simple charge transfer model in which $\mathrm{NO}_{2}$ molecules accept electrons from the CNT, thus increasing the hole population, while the $\mathrm{NH}_{3}$ molecules donate electrons, thus depleting the hole population and the conductance. However, such a conclusion for $\mathrm{NH}_{3}$ is inconsistent with theoretical results $[78,79]$ that $\mathrm{NH}_{3}$ molecules interact very weakly with pure CNTs, which would make charge transfer very difficult to explain.

A logical resolution to the above problem would be to note that CNTs are likely to have some defects incorporated either thermally or during high-temperature growth conditions. Also, they are not in isolation, and surrounding environment (oxygen, water vapor), or the substrate, or metal contacts at the ends might directly or indirectly provide a mechanism of binding of the gas molecules. In this section, we report $\mathrm{DMol}^{3}$ results on the chemisorption of a $\mathrm{NH}_{3}$ molecule on structural defects on a CNT. Fig. 6 displays dissociated $\mathrm{NH}_{2}$ and $\mathrm{H}$ fragments chemisorbed on a $(8,0)$ CNT containing various types of defects: (a) pristine CNT; (b) a vacancy (V); (c) an interstitial (I); (d) a Stone-Wales (SW) defect [80]; and (e) an $\mathrm{O}_{2}$ molecule pre-dissociated into a SW defect (SW_O_O). Table 1 displays the reaction energetics $\left(\Delta E_{\text {reac }}\right)$, activation barrier $\left(\Delta E_{\text {act }}\right)$, and net electron transfer $(\Delta q)$ from $\mathrm{NH}_{2}$ and $\mathrm{H}$ groups to the $\mathrm{CNT}$ for the five structures described by Fig. 6(a-e). We follow the convention that $\Delta E_{\text {reac }}<0$ for an exothermic process. The important results can be summarized as follows: 
i) Chemisorption to a defect-free $\mathrm{CNT}$ is an endothermic process with a large activation barrier, and therefore highly unlikely even at elevated temperatures.

ii) At both $\mathrm{V}$ and I the dissociation becomes exothermic with energy gains of 2.49 and $2.26 \mathrm{eV}$ respectively. The activation barrier for dissociation is rather low at $\mathrm{V}$, and should happen readily at room temperature, while that at $\mathrm{I}$ is also possible, although at a slower rate.

iii) At SW the dissociation is marginally exothermic, and the activation barrier is lower than that for a defect-free tube, although a bit high for chemisorption to happen readily at room temperatures. However, the presence of pre-dissociated O ( $\left.\mathrm{SW}_{-} \mathrm{O}_{-} \mathrm{O}\right)$ significantly enhances the stability of chemisorbed $\mathrm{NH}_{3}$ and makes the $\mathrm{NH}_{3}$ dissociation process nearly spontaneous.

iv) In all cases there is net electron transfer from the chemisorbed $\mathrm{NH}_{3}$ to the $\mathrm{CNT}$. As compared to the defect-free tube, the amount of charge transfer to V increases almost two-and-ahalf fold, while that to SW_O_O is enhanced nearly seven-fold. It is also to be noted here that multiple $\mathrm{O}_{2}$ molecules could potentially dissociate on the same SW defect, thus providing dissociation sites for multiple $\mathrm{NH}_{3}$ molecules, leading to much higher net charge transfer to the SWNT.

v) Computed infrared (IR) spectrum of some of the defect structures of Fig. 6 provides proper interpretation of recent FTIR data [81]. See ref. [82] for more details.

Large charge transfer should qualitatively explain the observed drops in electrical conductance, although a quantitative comparison would require explicit electronic transport calculations. Just to appreciate the complexity of the problem, we have plotted the electronic density of states (DOS) for the defect-free tube and for the structures in Fig. 6(e) and 6(b) respectively in Figs. 7(a-c). The DOS plot of the defect-free pristine tube (Fig. 7a) shows a bandgap of 0.022 Hartree $(=0.60 \mathrm{eV})$, in good agreement with previous DFT calculations [83]. Fig. 7(b) and 7(c) clearly demonstrate substantial modification to the DOS of Fig. 7(a), including splitting of states, introduction of new mid-gap states, and so on. The changes are due to a combined effect of the topological defects and that of the chemisorbed $\mathrm{NH}_{3}$, and a more detailed analysis is necessary to isolate the effects due to various factors. For the specific case of Fig. 7(c), we can see that the LUMO arises out of an 
orbital primarily localized on the chemisorbed species at the vacancy (Fig. 7d). Such states are expected to significantly impact electronic conductance through the carbon nanotube.

Predicting stable chemical structures upon adsorbate dissociation and computing the electronic structure are only half of the puzzle. In order to truly predict the working of a sensor device, it is necessary to compute electronic transport and the resulting electrical conductance through the nanotube. To this end, we are studying transport properties of molecules chemisorbed on CNTs using a multiscale approach involving DFT, the density functional based tight binding (DFTB) $[84,85]$ and NEGF methods. A DFT code (like DMol $\left.{ }^{3}\right)$ can generate optimized structures, which are rather limited in size with only one or two adsorbates. Using these structures as a template, we construct longer structures, which form the input to DFTB calculations that can handle many hundreds of atoms and is hence used to obtain the overlap and Hamiltonian matrices. Longer structures allow us to study sensor response as a function of defect dilution, an important consideration in the practical design and deployment of sensors. To gain further confidence in this multiscale approach we compared the DFTB- and DMol ${ }^{3}$-computed charge transfer between nanotube and adsorbates, and found quite good agreement.

As the dimension of the overlap and Hamiltonian matrices is in thousands, we exploit the onedimensionality of nanotubes to reconstruct the overlap and Hamiltonian matrices in a banded form by including more than nearest neighbor interaction. The diagonal blocks of this banded matrix typically correspond to a few rings of the underlying nanotube and adsorbates associated with these rings. This banded matrix is the input to our Green's function code to compute the transmission probability, density of states and charge density [86].

The change in current flowing through nanotubes due to gas adsorbtion could be due to a variety of reasons: doping of nanotube bulk, modulation of the Schottky barrier between metal and nanotube, and change in transmission due to defect-induced scattering. There have been many studies of the role of doping using DFT calculations on periodic systems and clusters [87-91]. The role of Schottky barrier modulation due to gases has also been phenomenologically studied [92, 93]. Our focus is on the adsorbate-induced change in the transmission function $T(E)$ and the 
density of states (DOS), and their dependence on the spatial density of adsorbates. One important aspect of the calculation is modeling of contacts. We currently treat the contacts either as semiinfinite ideal CNT leads (as described in section 4.1) or as a metal with featureless density of states. While such a model will not capture the exact nature of bonding between metal contact and nanotube atoms in experimental situations, it is sufficient for studying changes in $T(E)$ induced by adsorbates.

\subsection{CNT-Polymer composites}

The fact that the inclusion of even small amounts of nanomaterial coupled with appropriate processing steps appears to significantly improve mechanical, elastic, thermal, electrical, and optical absorpion properties have catapulted nanocomposites to being one of the first practical application areas of nanotechnology. One specific class of composite materials has recently received a lot of attention, i.e., in which carbon nanotubes (CNTs) are dispersed within polymeric matrices [4-6]. Potential applications can range from structural materials, to electromagnetic and heat shielding, to optoelectronics. The physical properties of CNT-polymer composite material depend on the uniformity of CNT dispersion and the degree of parallel alignment within the polymeric matrix, as well as the efficacy of interfacial bonding between the two systems. Since it is difficult to control many of these properties experimentally, modeling and simulations could provide crucial insight and design guidance.

One of the biggest challenges to CNT-based nanocomposites is that nanotubes like to form bundles, and they are difficult to disperse uniformly within the composite material. In a CNTpolymer composite, one would typically break up the CNT-bundle by supersonication, and then hope that each individual CNT is coated by polymer (see Fig. 8(a)), which prevents re-bundling of the CNTs. Successful dispersion using this strategy has been demonstrated in a few cases, most notably the modified Poly(p-phenylene vinylene) polymer (PmPV), which is able to disperse CNTs of a narrow diameter range, between 1.35 and $1.55 \mathrm{~nm}$ [94]. For successful dispersion, one clearly needs strong binding between the polymer and the CNT that can overcome strong van der 
Waals interaction between the tubes themselves. One could obtain some useful information on this binding through classical molecular mechanics (MM) simulations on small segments of individual CNTs, as we have done using the COMPASS force field [27] on structures like Fig. 8(b), where chiral $(12,7)$ and $(13,6)$ CNTs were studied as specific examples [95].

However, the smallest size and time-scale to describe the morphology of CNT-polymer nanocomposites and its dynamical evolution is currently beyond the capability of standard classical force field simulations, and it is necessary to take recourse to more coarse-grained approaches. For this we have used a particle-based mesoscale approach, in which the polymer and the CNT are represented as strings of beads, connected by Hookean springs, and dissipative particle dynamics (DPD) [34] is used to hydrodynamically equilibrate such coarse-grained morphology.

The chemistry of polymer-nanotube interaction is incorporated through relating the DPD beadbead repulsion to the Flory-Huggins $\chi$-parameter [37, 38], which in turn is obtained by squaring the difference of pure-component solubility parameters $(\delta)$ [96]. The latter is defined as the square root of the cohesive energy density. Reliable average values of $\delta$ for long-chain polymers can be estimated from simple correlation methods [97]. On the other hand, CNTs are not polymers in the conventional sense. Nevertheless, since CNTs tend to form close-packed bundles, a good measure of their cohesive energy can be obtained from the the energy cost of isolating a CNT from a bundle. Computing such energy with the Universal force field [28] results in $\delta$ that is essentially independent of the CNT chiral angle, and that decreases as inverse square-root of the CNT diameter, as illustrated in Fig. 9. Flory-Huggins theory predicts that components with close enough $\delta$, which leads to small DPD repulsion, should mix, while components with significantly differing $\delta$ should segregate. Thus, it follows from Fig. 9 that PMMA polymers should mix well with CNTs of diameters close to $1.4 \mathrm{~nm}$ (e.g. $(10,10)$ CNTs) (see Fig. 10(a)), but not with $(15,15)$ CNTs (diameters 1.9 nm), see Fig. 10(b). The CNT solubility parameter curve of Fig. 9 explains why PmPV polymers show selective affinity to CNTs of diameters $\sim 1.5 \mathrm{~nm}[94]$. 
Through DPD simulations we are exploring several important effects: (i) parallel alignment of the CNTs through an externally applied dynamical shear [40]; (ii) improvement in dispersion by functionalizing CNTs [40]; (iii) effect on the morphology through reduced dimensionality; (iv) critical CNT concentration for creating percolation networks; (v) employing diblock and triblock copolymers for creating novel self-assembled patterns. All of the above could be studied as a function of CNT and polymer lengths, relative compositions, shear rates, and the attached functional groups. As a final step, the resultant mesoscale morphologies are being represented on a numerical grid, and important properties like electrical and thermal conductivities are being computed using a finite-elements-based code [42-44].

\section{Challenges and future outlook}

Through a few application examples with carbon nanotubes we have illustrated the use of a variety of theoretical techniques spanning a wide spectrum of length and time-scales. As better synthesis and manipulation techniques for CNTs emerge, more complex and newer applications will be proposed, which would provide exciting opportunities for theory, modeling and simulations. However, several challenges, both experimental and theoretical, remain as a roadblock to successful commercial deployment of most technologies. As with any nanosystem, contact remains a critical issue. Small atomic-level changes in the structure of the contact can have a significant impact on the contact resistance, and very little characterization data exists on most experimental contacts. Besides, even though DFT-based NEGF codes are becoming faster and more accurate, they are still too limited in realistically representing metal-CNT contacts. In CNTpolymer composites, even if the CNTs are well dispersed one would need to control and study the mechanical, electrical and thermal coupling between polymer and CNT in much more detail than has been afforded to date. Standard DFT or tight-binding treatments, as described here, also does not take into account complex many-body electron-correlation effects that may arise at low temperatures, or Coulomb blockade effects when electrons in CNTs get highly localized due to large mechanical deformation or highly resistive contacts. For some applications it may also 
become necessary to design a truly multiscale code, i.e., one that integrates multiple codes developed to address different length and time scales, limited examples of which exist in the literature [98-100].

Acknowledgements: The author would like to acknowledge collaborations with M. P. Anantram, A. Svizhenko, A. Ricca, J. Andzelm, J. Wescott, G. Goldbeck-Wood, P. Kung, M. in-het Panhuis, and W. Blau. The work was performed under the auspices of the U.S. Department of Energy by the University of California Lawrence Livermore National Laboratory under Contract W-7405Eng-48. 


\section{References}

1. S. Iijima, Nature 354 (1991) 56.

2. Articles on nanotubes in Physics World 13, Issue 6 (2000) pp. 29-53.

3. M.Terrones, Annual Review of Materials Research 33 (2003) 419-501.

4. R. H. Baughman, A. A. Zakhidov, and W. A. de Heer, Science 297 (2002) 787.

5. P. M. Ajayan and O. Zhou, in "Carbon Nanotubes Synthesis, Structure, Properties and Applications," (eds. Dresselhaus, M. S.; Dresselhaus, G.; Avouris, P.) Pg. 391-425, SpringerVerlag (2001).

6. M. Meyyappan (ed.), "Carbon Nanotubes - Science and Applications," CRC Press (2004).

7. J. W. Mintmire, B. I. Dunlap, and C. T. White, Phys. Rev. Lett. 68 (1992) 631.

8. N. Hamada, S. Sawada, and A. Oshiyama, Phys. Rev. Lett. 68 (1992) 1579.

9. R. Saito, M. Fujita, G. Dresselhaus, and M. S. Dresselhaus, Appl. Phys. Lett. 60 (1992) 2204.

10. Technical Proceedings of the 2003 Nanotechnology Conference and Trade Show, Volume 3, NSTI Publications, Cambridge, MA (2003).

11. Technical Proceedings of the 2002 International Conference on Computational Nanoscience and Nanotechnology, Volume 2, NSTI Publications, Cambridge, MA (2002).

12. Technical Proceedings of the 2001 International Conference on Computational Nanoscience and Nanotechnology, Volume 2, NSTI Publications, Cambridge, MA (2001).

13. J. Bernholc, D. Brenner, M. B. Nardelli, V. Meunier, and C. Roland, Annual Review of Materials Research 32 (2002) 347-375.

14. D. Tománek and R. Enbody (eds.), Science and Applications of Nanotubes, Kluwer Academic Publishers, Netherlands (2000).

15. Articles in Physica B: Condensed Matter 323, No. 1-4 (October, 2002). 
16. M. S. Dresselhaus, G. Dresselhaus, and Ph. Avouris (eds.), "Carbon Nanotubes - Synthesis, Structure, Properties, and Applications," Springer (2001).

17. S. Reich, C. Thomsen, and J. Maultzsch, "Carbon Nanotubes - Basic Concepts and Physical Properties," Wiley-VCH (2004).

18. J. A. Schwarz, C. I. Contescu, and K. Putyera (eds.) "Dekker Encyclopedia of Nanoscience and Nanotechnology," Mercel Dekker (2004).

19. F. Jensen, "Introduction to Computational Chemistry,” John Wiley and Sons (1999).

20. A. Szabo and N. S. Ostlund, "Modern Quantum Chemistry," Dover Publications (1996).

21. R. G. Parr and W. Yang, "Density Functional Theory of Atoms and Molecules," Oxford University Press (1989).

22. P. Hohenberg and W. Kohn, Phys. Rev. B 136 (1964) 864.

23. W. Kohn and L. J. Sham, Phys. Rev. A 140 (1965)1133.

24. B. Delley, J. Chem. Phys. 92 (1990) 508; J. Phys. Chem. 100 (1996) 6107; Int. J. Quantum Chem. 69 (1998) 423; J. Chem. Phys. 113 (2000) 7756.

25. J. P. Perdew, K. Burke, M. Ernzerhof, Phys. Rev. Lett. 77 (1996) 3865.

26. J.-R. Hill, L. Subramanian, and A. Maiti, "Molecular Modeling Techniques in Material Sciences," CRC Press, Taylor and Francis (2005).

27. H. Sun, J. Phys. Chem. B 102 (1998) 7338.

28. A. K. Rappe, C. J. Casewit, K. S. Colwell, W. A. Goddard, and W. M. Skiff, J. Am. Chem. Soc. 114 (1992) 10024.

29. S. Datta, “Quantum Transport: Atom to Transistor," Cambridge University Press (2005).

30. S. Datta, "Electronic Transport in Mesoscopic Systems," Cambridge University Press (1997).

31. Y. Imry, "Introduction to Mesoscopic Physics," Oxford University Press (1997). 
32. D. K. Ferry and S. M. Goodnick, "Transport in Nanostructures," Cambridge University Press (1997).

33. C. W. J. Beenakker, Rev. Mod. Phys. 69 (1997) 731.

34. R. D. Groot and P. B. Warren, J. Chem. Phys. 107 (1997) 4423.

35. C. M. Wijmans, B. Smit, and R. D. Groot, J. Chem. Phys. 114 (2001) 7644.

36. A. Maiti and S. McGrother J. Chem. Phys. 120 (2004) 1594.

37. P. J. Flory, "Principles of Polymer Chemistry", Cornell University Press (1953).

38. M. Doi, "Introduction to Polymer Physics," Chapter 2, Clarendon Press, Oxford (1996).

39. P. Espaňol and P. B. Warren, Europhys. Lett. 30 (1995) 191.

40. A. Maiti, J. Wescott, and P. Kung, Mol. Sim. 31 (2005) 143.

41. A. Maiti, J. Wescott, and G. Goldbeck-Wood, Int. J. Nanotechnology 2 (2005) 198.

42. A. A. Gusev, Macromolecules 34 (2001) 3081.

43. http://www.matsim.ch/MedienE.html.

44. http://www.accelrys.com/products/mstudio/modeling/polymersandsimulations/mesoprop.html

45. P. R. Wallace, Phys. Rev. 71 (1947) 622.

46. C. T. White, D. H. Robertson, and J. W. Mintmire, Phys. Rev. B 47 (1993) 5485.

47. R. A. Jishi, D. Inomata, K. Nakao, M. S. Dresselhaus and G. Dresselhaus, J. Phys. Soc. Jpn. 63 (1994) 2252.

48. C. T. White, J. W. Mintmire, R. C. Mowrey, D. W. Brenner, D. H. Robertson, J. A. Harrison, and B. I. Dunlap, in Buckminsterfullerenes, edited by W. E. Billups and M. A. Ciufolini, VCH Publishers, NY (1993).

49. S. Barber, Y. K. Kwon, and D. Tomanek, Phys. Rev. Lett. 84 (2000) 4613. 
50. T. W. Tombler, C. Zhou, L. Alexseyev, J. Kong, H. Dai, L. Liu, C. S. Jayanthi, M. Tang, S. Y. Wu, Nature 405 (2000) 769.

51. M. Nardelli and J. Bernholc, Phys. Rev. B 60 (1999) R16338.

52. A. Rochefort, P. Avouris, F. Lesage, and D. Salahub, Phys. Rev. B 60 (1999) 13824.

53. L. Liu, C. S. Jayanthi, and H. Dai, Phys. Rev. Lett. 84 (2000) 4950.

54. A. Maiti, Phys. Stat. Sol. B 226 (2001) 87.

55. D. A. Papaconstantopoulos, M. J. Mehl, S. C. Erwin and M. R. Pederson, Tight-Binding Approach to Computational Materials Science, P.E.A. Turchi, A. Gonis, and L. Colombo, eds., MRS Proceedings 491, (Materials Research Society, Warrendale, PA, 1998)

56. A. Maiti, A. Svizhenko, and M. P. Anantram, Phys. Rev. Lett. 88 (2002) 126805.

57. C. L. Kane and E. J. Mele, Phys. Rev. Lett. 78 (1997) 1932.

58. R. Heyd, A. Charlier, and E. McRae, Phys. Rev. B 55 (1997) 6820.

59. L. Yang, M. P. Anantram, J. Han, and J. P. Lu, Phys. Rev. B 60 (1999) 13874.

60. L. Yang and J. Han, Phys. Rev. Lett. 85 (2000) 154.

61. A. Kleiner and S. Eggert, Phys. Rev. B 63 (2001) 073408.

62. P. E. Lammert, P. Zhang, and V. H. Crespi, Phys. Rev. Lett. 84 (2000) 2453.

63. J-Q. Lu, J. Wu, W. Duan, F. Liu, B. F. Zhu, and B. L. Gu, Phys. Rev. Lett. 90 (2003) 156601.

64. A. Svizhenko, H. Mehrez, M. P. Anantram, and A. Maiti, Proc. SPIE 5593 (2005) 416.

65. E. D. Minot, Y. Yaish, V. Sazonova, J-Y. Park, M. Brink, and P. L. McEuen, Phys. Rev. Lett. $90(2003) 156401$.

66. J. Cao, Q. Wang, and H. Dai, Phys. Rev. Lett. 90 (2003) 157601.

67. A. Maiti, Nature Materials(London) 2 (2003) 440. 
68. Y. Zhang, N. W. Franklin, R. J. Chen, and H. Dai, Chem. Phys. Lett. 331 (2000) 35.

69. D. Mann, A. Javey, J. Kong, Q. Wang, H. Dai, Nano Lett. 3 (2003) 1541.

70. A. Javey, J. Guo, Q. Wang, M. Lundstrom, H. Dai, Nature 424 (2003) 654.

71. E. Durgun, S. Dag, V. M. K. Bagci, O. Gülseren, T. Yildirim, S. Ciraci, Phys. Rev. B 67, (2003) 201401.

72. A. Maiti and A. Ricca, Chem. Phys. Lett. 395 (2004) 7.

73. J.Kong, N.R.Franklin, C.Zhou, M.G.Chapline, S.Peng, K.Cho and H.Dai, Science 287 (2000) 622.

74. P.G.Collins, K.Bradley, M.Ishigami and A. Zettl, Science 287 (2000) 1801.

75. P. Qi, O. Vermesh, M.Grecu, A. Javey, Q. Wang, H.Dai, S. Peng, K.Cho, Nano Lett. 3 (2003) 347.

76. L. Valentini, I. Armentano, J.M. Kenny, C.Cantalini, L.Lozzi and S.Santucci, Appl. Phys.Lett. 82 (2003) 961.

77. S. Chopra, A.Pham, J. Gaillard, A.Parker and M.Rao, Appl.Phys. Lett. 80 (2002) 4632.

78. H.Chang. J.D.Lee, S.M.Lee and Y.H.Lee, Appl.Phys.Lett. 79 (2001) 3863.

79. J. Zhao, a. Buldum, J. Han and J.P. Lu, Nanotechnology 13 (2002) 195.

80. A.J. Stone and D.J. Wales, Chem.Phys.Lett. 128 (1986) 501.

81. M.D. Ellison, M.J.Crotty, D. Koh, R.L. Spray, K.E. Tate, J. Phys. Chem. B 108 (2004) 7938.

82. J. Jandzelm, N. Govind, and A. Maiti, Chem. Phys. Lett. (2006) in press.

83. X. Blase, L. Benedict, E. L. Shirley, and S. G. Louie, Phys. Rev. Lett. 72 (1994) 1878.

84. M. Elstner, D. Porezag, G. Jungnickel, J. Elsner, M. Haugk, T. Frauenheim, S. Suhai, and G. Seifert, Phys. Rev. B 58 (1998) 7260.

85. T. Frauenheim, G. Seifert, M. Elstner, Z. Hajnal, G. Jungnickel, D. Porezag, S. Suhai, and R. Scholz, Phys. Stat. Sol.(b) 217 (2000) 41. 
86. A. Svizhenko, M. P. Anantram, T. R. Govindan, B. Biegel and R. Venugopal, J. Appl. Phys. $91(2002) 2343$.

87. S.-H. Jhi, S. G. Louie, and M. L. Cohen, Phys. Rev. Lett. 85 (2000) 1710.

88. D. C. Sorescu, K. D. Jordan, and Ph. Avouris, J. Phys. Chem. 105 (2001) 11227.

89. J. Zhao, A. Buldum, J. Han, and J. P. Lu, Nanotechnology 13 (2002) 195.

90. A. Ricca and J. A. Dorocco, Chem. Phys. Lett. 367 (2002) 217.

91. P. Giannozzi, R. Car, and G. Scoles, J. Chem. Phys. 118 (2003) 1003.

92. R. Martel, V. Derycke, J. Appenzeller, K. K. Chan, J. Tersoff, and Ph. Avouris, Phys. Rev. Lett. 87 (2001) 256805.

93. S. Heinze, J. Tersoff, R. Martel, V. Derkcke, J. Appenzeller, and Ph. Avouris, Phys Rev. Lett 89 (2002) 106801.

94. A. B. Dalton, C. Stephan, J. N. Coleman, B. McCarthy, P. M. Ajayan, S. Lefrant, P. Bernier, W. J. Blau, and H. J. Byrne, J. Phys. Chem. B 104 (2000) 10012.

95. M. in het Panhuis, A. Maiti, A. B. Dalton, A. van den Noort, J. N. Coleman, B. McCarthy, and W. J. Blau, J. Phys. Chem. B 109 (2003) 478.

96. F. Case and J. D. Honeycutt, Trends in Polymer Science 2 (1994) 259.

97. J. Bicerano, "Prediction of Polymer Properties," 2nd Ed, Mercel Dekker (1996) Pg 108-136. The polymer solubility parameters of Fig. 9 were computed as an average of the Fedors and van Krevelen solubility parameters as defined in this reference.

98. R. E. Rudd and J. Q. Broughton, J. Modeling and Sim. Of Microsys. 1 (1999) 29.

99. F. F. Abraham, J. Q. Broughton, N. Bernstein, and E. Kaxiras, Europhys. Lett. 44 (1998) 783.

100.R. E. Rudd and J. Q. Broughton, Phys. Stat. Sol. B 217 (2000) 251. 
Table 1. $\mathrm{NH}_{3}$ dissociation (into $\mathrm{NH}_{2}$ and $\left.\mathrm{H}\right)$ on a $(8,0) \mathrm{CNT}$ : reaction energetics $\left(\Delta \mathrm{E}_{\text {reac }}\right.$ ), activation barrier $\left(\Delta \mathrm{E}_{\mathrm{act}}\right)$, and net electron transfer $(\Delta \mathrm{q})$ from $\mathrm{NH}_{2}$ and $\mathrm{H}$ groups to the CNT. Negative values of $\left(\Delta \mathrm{E}_{\text {reac }}\right)$ denotes an exothermic process.

\begin{tabular}{|c|c|c|c|c|}
\hline Substrate & $\begin{array}{c}\text { Resulting bonding } \\
\text { configuration on CNT surface }\end{array}$ & $\begin{array}{c}\Delta \mathrm{E}_{\text {reac }} \\
(\mathrm{eV})\end{array}$ & $\begin{array}{l}\Delta \mathrm{E}_{\text {act }} \\
(\mathrm{eV})\end{array}$ & $\begin{array}{l}\Delta \mathrm{q} \text { from } \\
\mathrm{NH}_{3}(\mathrm{el})\end{array}$ \\
\hline CNT (defect-free) & $\begin{array}{l}\mathrm{C}_{3}-\mathrm{NH}_{2}+\mathrm{C}_{3}-\mathrm{H} \\
\text { (Fig. 6a) }\end{array}$ & +0.77 & 2.38 & 0.025 \\
\hline $\mathrm{V}$ & $\begin{array}{c}\mathrm{C}_{2}-\mathrm{NH}_{2}+\mathrm{C}_{2}-\mathrm{H} \\
\text { (Fig. 6b) }\end{array}$ & -2.49 & 0.35 & 0.063 \\
\hline $\mathrm{I}$ & $\begin{array}{l}\mathrm{NH}_{2}-\mathrm{C}_{2} \text {, bridge }-\mathrm{H} \\
\quad \text { (Fig. 6c) }\end{array}$ & -2.26 & 1.13 & 0.036 \\
\hline SW1 & $\begin{array}{l}\mathrm{C}_{3,577}-\mathrm{NH}_{2}+\mathrm{C}_{3,577}-\mathrm{H} \\
\quad \text { (Fig. 6d) }\end{array}$ & -0.17 & 1.50 & 0.044 \\
\hline SW1_O_O & $\begin{array}{l}\mathrm{C}_{3,577,}, \mathrm{o}^{-\mathrm{NH}_{2}}+\mathrm{C}_{3,577}-\mathrm{O}-\mathrm{H} \\
\text { (Fig. 6e) }\end{array}$ & -2.77 & 0.25 & 0.176 \\
\hline
\end{tabular}

$\mathrm{C}_{3}=$ Regular 3-fold coordinated $\mathrm{sp}^{2}$ carbon on a defect-free CNT; $\mathrm{C}_{2}=\mathrm{C}_{3}$ atom with a missing $\mathrm{C}$ neighbor; $\mathrm{C}_{3,577}=\mathrm{sp}^{2}$ carbon at a SW1 site shared by two heptagons and a pentagon; $\mathrm{C}_{3,577, \mathrm{O}}=\mathrm{C}_{3,577}$ atom with a bridging $\mathrm{O}$ separating it from one of its $\mathrm{C}$ neighbors 
Figure captions:

Figure 1. Carbon nanotube (CNT) basics. (a) A graphite sheet with lattice vectors $\boldsymbol{a}_{1}, \boldsymbol{a}_{2}$. A few lattice points are indicated, as is the chiral angle $\theta$ for a $(3,1)$ CNT. Dotted and dashed lines are drawn along circumferences of armchair and zigzag tubes respectively; (b) a (5, 5) armchair tube; (c) a (9, 0) zigzag tube.

Figure 2. Schematic showing stretching of a nanotube upon AFM-deformation.

Figure 3. (a) AFM deformation of a $(6,6)$ tube by a Li-needle. Respective deformation angles are indicated. Also indicated are the QM and the MM regions. (b) QM clusters at $25^{\circ}$ of deformation showing no signs of $\mathrm{sp}^{3}$ coordination.

Figure 4. Computed electrical conductance for $(12,0) \mathrm{CNT}$ - comparison between AFM-deformed and uniformly stretched tubes. Inset displays density of states plot for the $(12,0)$ tube at the largest deformations, showing opening of a bandgap at the Fermi energy. The figure also displays the conductance of a $(36,0)$ tube subjected to a uniform stretch.

Figure 5. (8, 0) SWNT on metal surfaces: (a) Au (100); (b) Pd (111); (c) Pt (111). On the Au surface the SWNT is essentially weakly physisorbed. For Pd and Pt surfaces, the metal-adjacent C-atoms undergo $\mathrm{sp}^{2} \rightarrow \mathrm{sp}^{3}$ transition, which significantly deform the CNT cross-section. Binding on Pd is slightly higher than on Pt.

Figure 6. $\mathrm{NH}_{3}$ dissociated at various defects on a $(8,0) \mathrm{CNT}$ : (a) defect-free tube; (b) vacancy; (c) interstitial; (d) a Stone-Wales (SW) defect; and (e) an $\mathrm{O}_{2}$ molecule pre-dissociated at a SW defect (SW_O_O). Dissociated $\mathrm{NH}_{2}$ and $\mathrm{H}$ fragments are shown in ball representation. In Fig. 6(e), the second $\mathrm{O}$ breaks an $\mathrm{O}-\mathrm{C}$ bond and creates a $\mathrm{OH}$ group single-bonded to the other $\mathrm{C}$-atom.

Figure 7. Density of States (electrons/Hartree) around Fermi energy for: (a) defect-free tube; (b) $\mathrm{NH}_{3}$ chemisorbed at SW_O_O (structure in Fig. 6(e)); and (c) chemisorbed $\mathrm{NH}_{3}$ at a vacancy (structure in Fig. 6(b)). (4) LUMO orbital of Fig. 7(c), showing localized state at the vacancy site. For Figs. 7(a-c), the energy unit (x-axis) is in Hartree. 
Figure 8. (a) Schematic showing how polymers can coat and debundle nanotubes; (b) Atomistic model of a PmPV polymer segment on a $(12,7)$ chiral nanotube relaxed using the COMPASS force field.

Figure 9. Plot of calculated solubility parameter $(\delta)$ (in units of $\left.\left(\mathrm{J} / \mathrm{cm}^{3}\right)^{1 / 2}\right)$ versus tube diameter for armchair (filled circles) and zigzag (unfilled diamonds) CNTs. The published [97] solubility values for poly(urethane) (PU), poly(styrene) (PS), poly(methylmethacrylate) (PMMA), poly(mphenylenevinylene) (PmPV), polyethylene (PE), polypropylene (PP) and poly(dimethylsiloxane) (PDMS) are also indicated on the plot for comparison.

Figure 10. Dispersion of nanotubes in PMMA polymer: (a) $(10,10)$ CNTs showing good dispersion; (b) $(15,15)$ CNTs showing poor dispersion and formation of CNT bundles. 


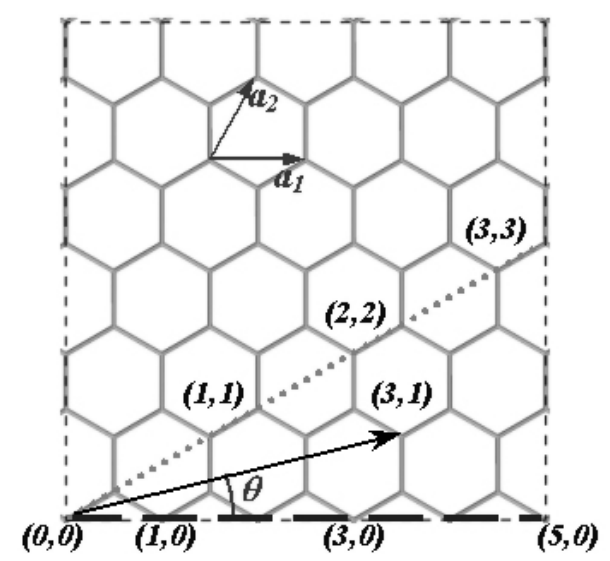

(a)

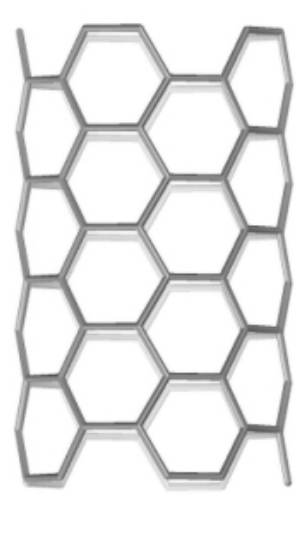

(b)

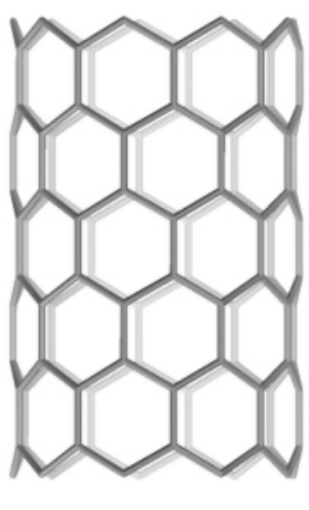

(c)

Figure 1 


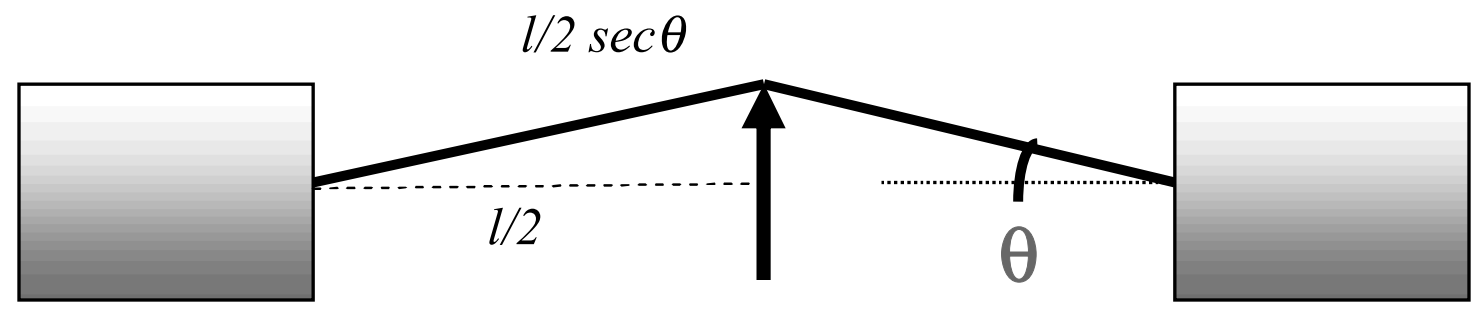

Figure 2 


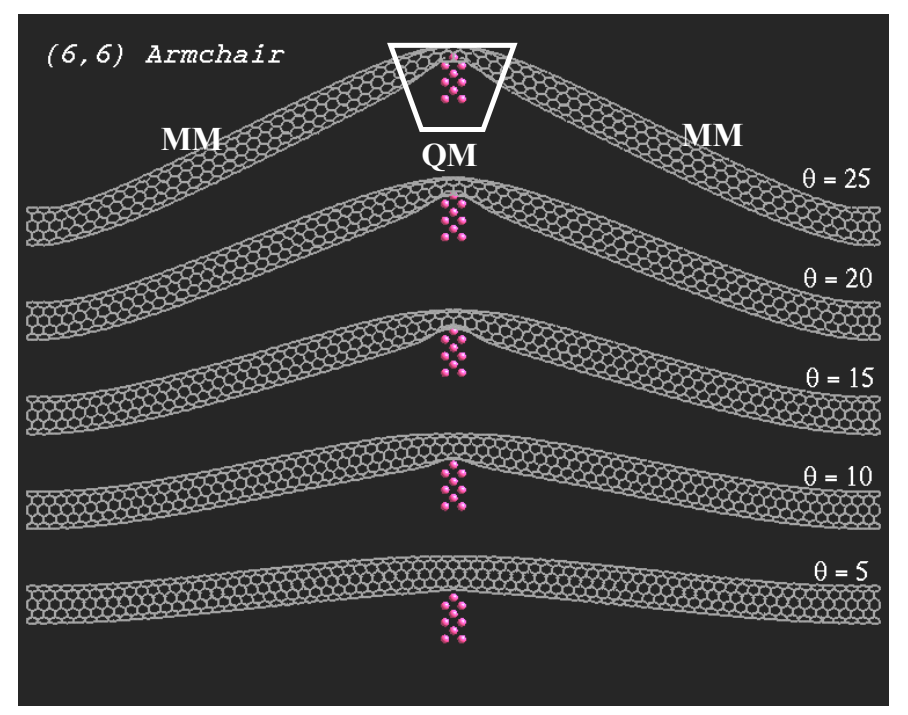

(a)

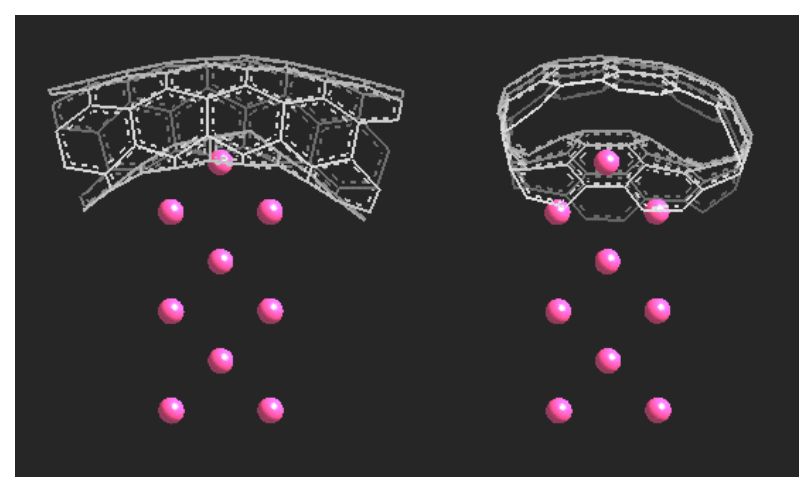

(b)

Figure 3 


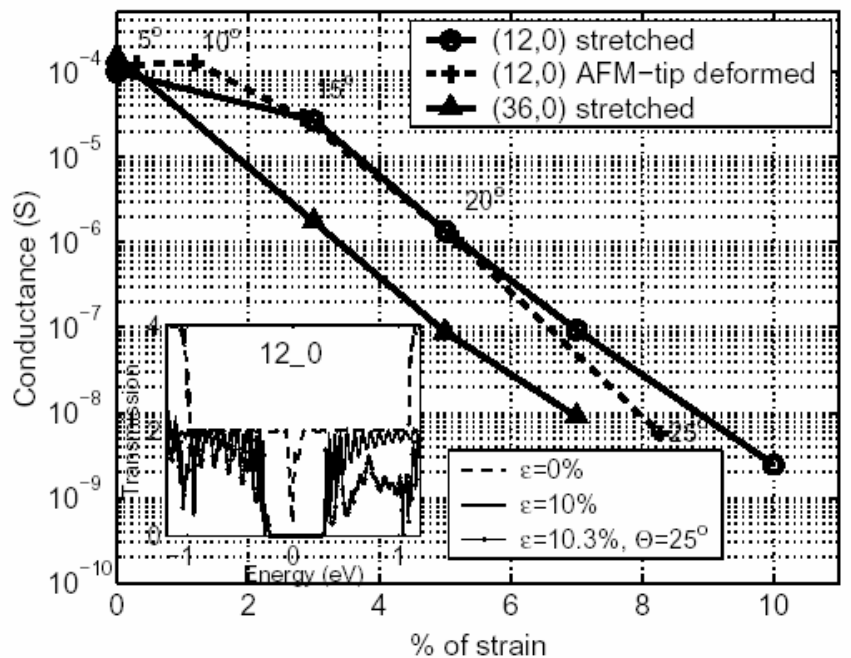

Figure 4 


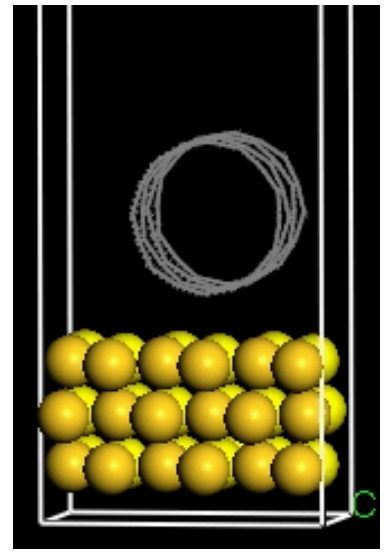

(a)

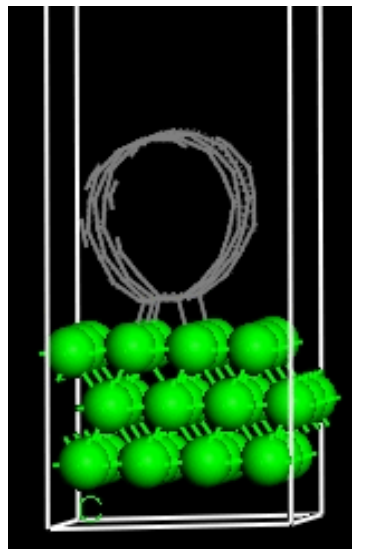

(b)

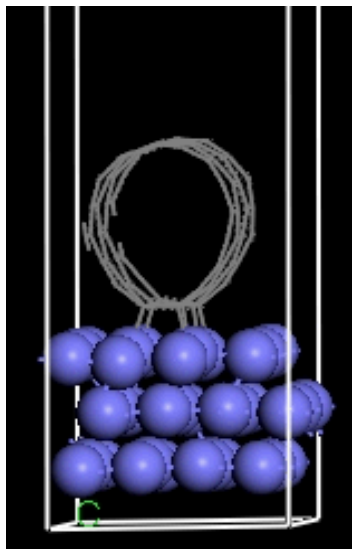

(c)

Figure 5 


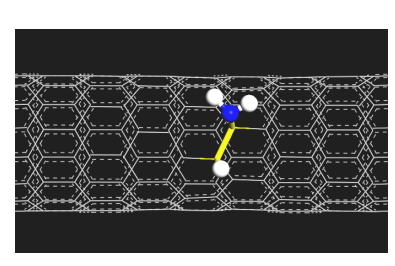

(a)

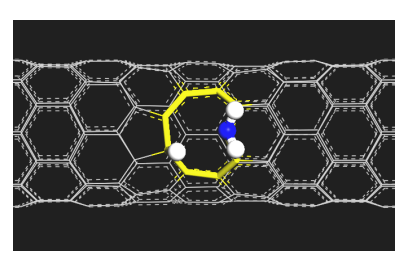

(b)

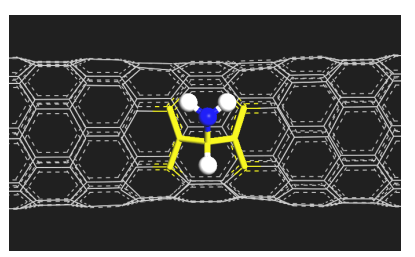

(c)

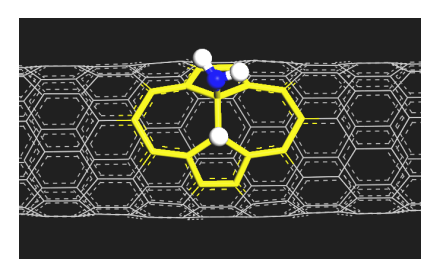

(d)

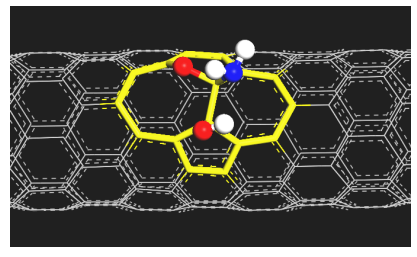

(e)

Figure 6 


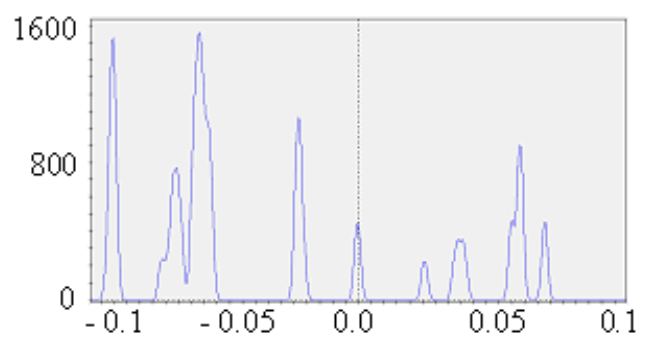

(a)

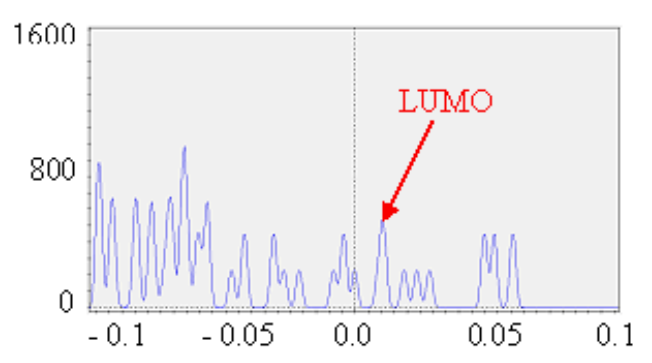

(b)

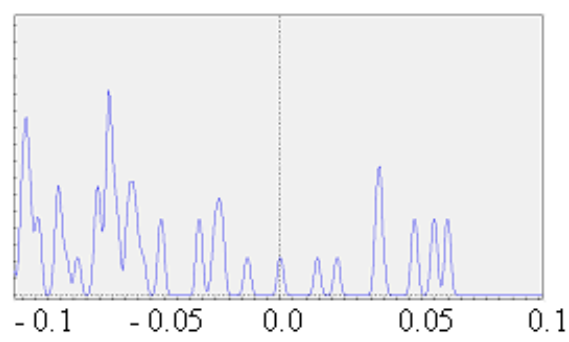

(b)

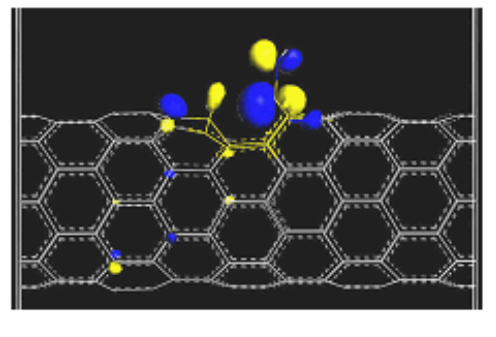

(d)

Figure 7 


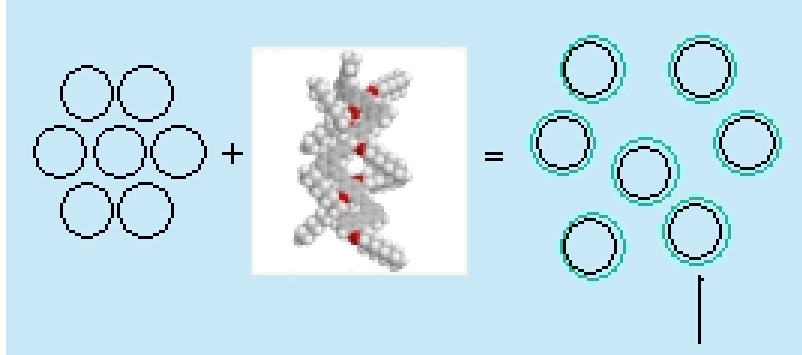

Polymer coated namotubes

(a)

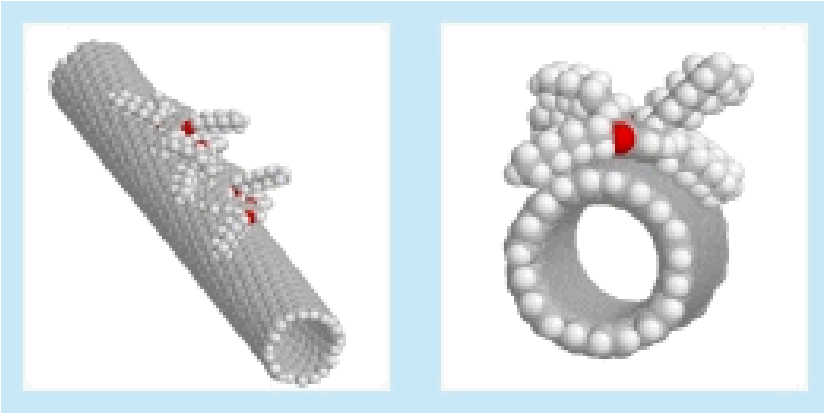

(b)

Figure 8 


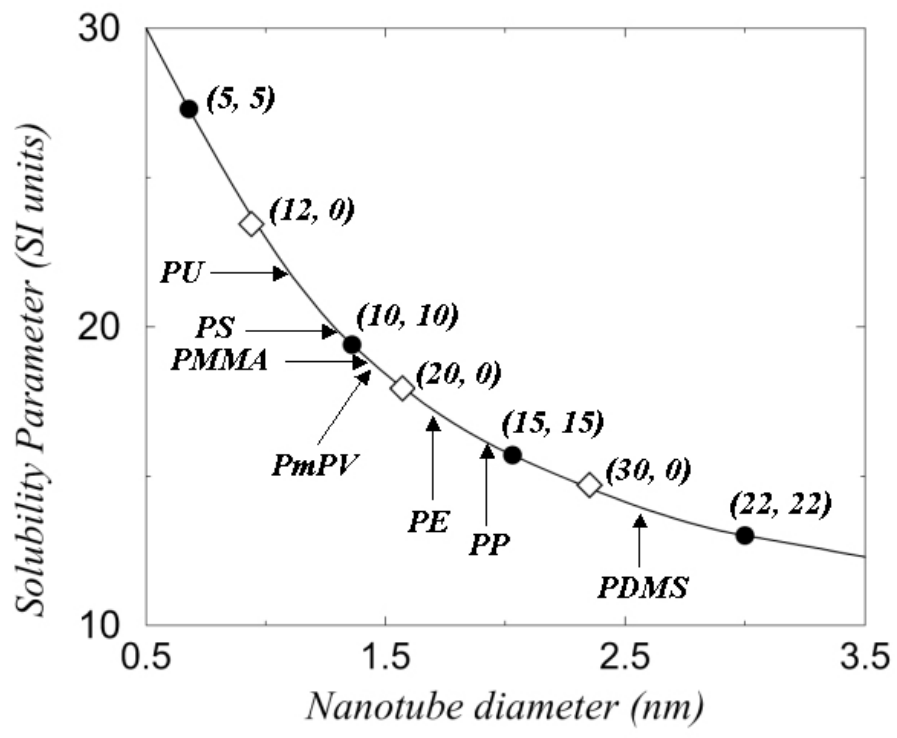

Figure 9 

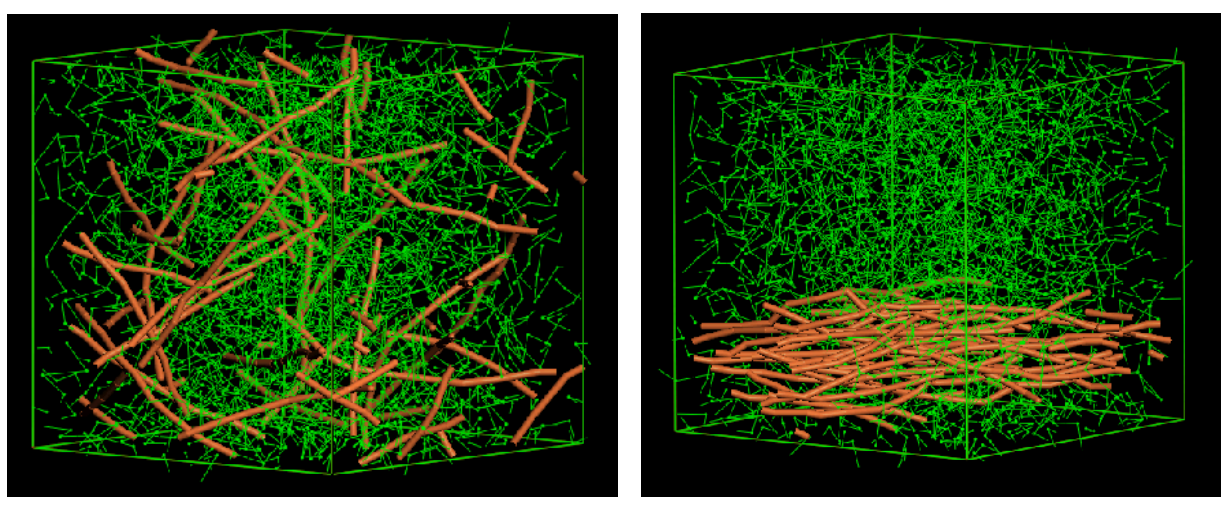

(a)

(b)

Figure 10 